\title{
A Novel Approach in Designing PID Controller for Semi-active Quarter Car Model
}

\author{
Vedant Mehta, Yash Gandhi, Mayuri Patel, Bhargav Gadhvi, Anil Markana, Jay J. Vora \\ Pandit Deendayal Petroleum University, Raisan, Gandhinagar - 382007, India
}

\begin{abstract}
This paper implements Teaching-Learning based optimization (TLBO) to obtain optimized value of spring stiffness for better ride comfort. Further, this optimized value is then used in a semi-active quarter car setup to remove any discrepancies due to non-optimized spring. This paper also introduces a novel approach to control the Semiactive suspension parameter (damping coefficient) for a better performance. For controlling semi-active parameters, PID controller has been used. PID controller output is fed to the quarter car setup as a damping coefficient. Thus changing the damping coefficient dynamically as the disturbance occurs, and thus improving the ride comfort. The sprung mass acceleration and rattle space of semi-active quarter car has been compared with sprung mass acceleration and rattle space of passive quarter car model to show the difference in results and thereby, results and conclusions are drawn.
\end{abstract}

\section{Introduction}

Various researches have been made in automotive industry to improve the suspension system. Suspension system is one of the most vital parts of any vehicle. It makes the vehicle ride smoother over rough roads having bumps and potholes. Designing of a suspension system is a compromise between vehicle handling and ride comfort. Sports car has good vehicle handling but doesn't provide good ride qualities, whereas, a sedan or a luxury car has outstanding ride comfort but low road handling quality.

Suspension system is classified as: passive, active and semi active.

Passive suspension system is the traditional suspension consisting of springs and dampers. A passive suspension uses spring to store energy and to dissipate it, a damper is used. Generally, its parameters are fixed and they are chosen in such a way that there is a certain level of compromise between road handling, load carrying and ride comfort [1].

An active suspension system is externally controlled and may vary its parameters. It contains an actuator (usually hydraulic, pneumatic or electromechanical) which creates force to control the motion of sprung mass and relative velocity between sprung and unsprung mass. This actuator is controlled with the help of a controller. Different control strategies are developed for the same. Active suspension system is a closed loop control system whereas passive suspension system is an open loop control system $[1,2]$.
Semi-active suspension system consists of a varying damper. The force applied by semi-active suspension system is created by changing the fluid flow of damper. Magnetorheological (MR) fluid damper with many advantages such as high viscosity control range, comparatively low cost, low power consumption and quick response, small size etc. is an excellent choice for semi active damper fluid [3].

Due to its low energy consumption with similar vibration control performance to the active control methods Semi-active control has shown many advantages in vehicle suspension systems [4]. Its ability to control the damping force is also a major advantage. A compromise between the simplicity of passive systems, and the cost of higher- performance fully active suspension system can be offered by Semi-active suspension system [5].

Over years different optimization techniques have been used for optimization of various parameters $[6,7]$. Bagheri-2011 [8] presented a paper in which a multiobjective genetic algorithm with a recently developed diversity preserving mechanism called as NSGA-II, has been used to optimally design vehicle vibration model.

Badran-2012 [9] worked on optimization of a fourdegrees-of-freedom vehicle's human with seat suspension system using genetic algorithms (GA) to determine vehicle suspension parameters to achieve the best comfort of the human. 
Qazi-2013[10] reached to result that fuzzy logic based particle swarm optimized semi-active suspension system is better than passive and active suspension model in terms of road handling and ride comfort. Particle swarm optimization was used to optimize scaling factors keeping normalized ranges of inputs and outputs of fuzzy logic controller.

Teacher learning based optimization (TLBO) was used to optimize parameters of PID integrated active suspension system. When optimized values of suspension stiffness and damping constants were compared with non-optimized, a $36 \%$ reduction in effort of actuator force and $41 \%$ reduction in suspension travel was observed [11].

In this paper, stiffness constant of sprung mass of passive suspension has been optimized using TLBO. For comparison purpose, a PID integrated semi-active suspension model has been used. Comparison between optimized and non-optimized values is done on the basis of rattle space and sprung mass acceleration.

\section{Optimization of passive quarter car model}

Passive quarter car model consists of sprung mass, unsprung mass, and suspension (consisting of spring and damper). Sprung mass consists of the upper body of the car including chassis weight. Unsprung mass consists of wheel assembly, and the parts below the suspension. Tire also has its own stiffness and damping properties. Stiffness of tire is very large and damping coefficient of tire is very negligible. Road input is given to tire and this input is transferred to unsprung mass and then to sprung mass via suspension. So sprung mass experiences less vibrations as compared to unsprung mass.

Figure shown below resembles the quarter car model.

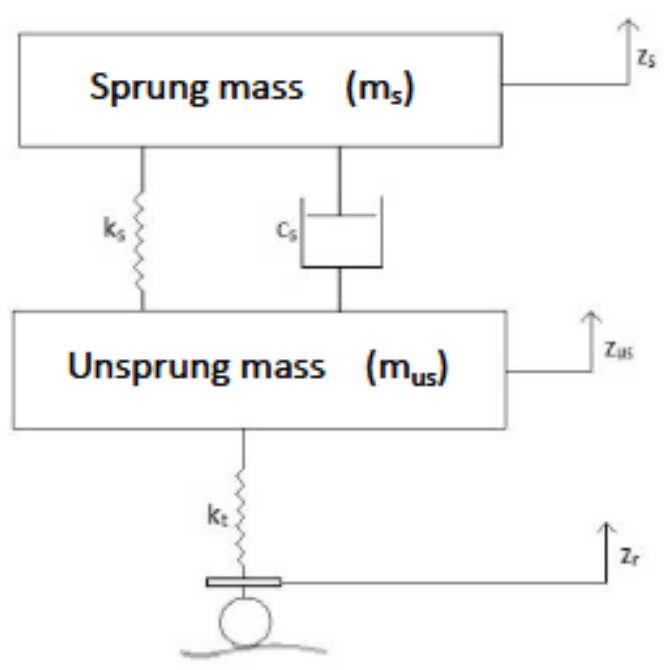

Figure 1: Quarter car model (Passive suspension system)
Optimizing the spring stiffness of the suspension helps in better ride comfort. Optimized value reduces the amplitude of vibrations felt by sprung mass and helps improve the damping of vibrations.

\subsection{Teaching learning based optimization (TLBO)}

A new optimization algorithm, called 'TeachingLearning-Based Optimization (TLBO) [10] is used for the optimization of passive suspension system parameters. This method works on the basis of types of interaction between a teacher and learner. TLBO is a populationbased method where it uses various solutions generated from each population to obtain a global solution.

This algorithm consists of two phases:

1. Teacher phase' which means interaction between teacher and a learner.

2. Teacher phase' which means interaction between teacher and a learner.

TLBO shows better performance as compared to other nature-inspired optimization algorithms such as Artificial Bee Colony (ABC), Particle Evolutionary Swarm Optimization (PESO), Cultural Differential Evolution (CDE), etc. for the constrained benchmark functions for different performance criteria, such as success rate, mean solution, average number of function evaluations required, convergence rate, etc. [12]. Different algorithms require their own algorithm-specific control parameters. The improper tuning of algorithm-specific parameters either increases the computational effort or yields the local optimal solution. Thus, TLBO proves to better as compared to other algorithms.

\subsection{Optimization using TLBO}

Since the design of suspension system is trade-off between ride comfort and road handling, this paper considers a weighted objective function consisting of ride comfort as well as road handling [13].

Objective function:

$$
y=r m s\left(\ddot{z}_{s}\right)+\left(1000 \times r m s\left(z_{u s}-z_{r}\right)\right.
$$

$\ddot{z}_{s}$ - represents sprung mass acceleration (ride comfort) $\left(z_{u s}-z_{r}\right)$ - represents tire deflection (road handling) Quarter car parameters are shown in the table below

Table 1. Quarter car parameters

\begin{tabular}{|c|c|c|}
\hline Symbol & Name & Value \\
\hline ms & Sprung mass & $327 \mathrm{~kg}$ \\
\hline mus & Un-sprung mass & $116 \mathrm{~kg}$ \\
\hline $\mathbf{k s}$ & $\begin{array}{c}\text { Suspension stiffness constant } \\
\text { (optimized) }\end{array}$ & $60186 \mathrm{~N} / \mathrm{m}$ \\
\hline $\mathbf{k t}$ & $\begin{array}{c}\text { Tire stiffness constant } \\
\text { cs }\end{array}$ & $\begin{array}{c}\text { Suspension damping coefficient } \\
\text { (non-optimized) }\end{array}$ \\
\hline
\end{tabular}




\section{Semi-active model and controller scheme}

In this paper, semi-active suspension system has been taken into consideration. Semi-active suspension system has a variable damping system. Magneto-rheological fluid (MRF) is used as a damping fluid. This fluid has magnetic properties. The viscosity of the fluid can be controlled by subjecting it to magnetic field. This feature helps improve ride comfort. The damping coefficient varies within a defined range. Thus, semi-active is a kind of adaptive system which adapts to the varying road and thus improves the ride comfort.

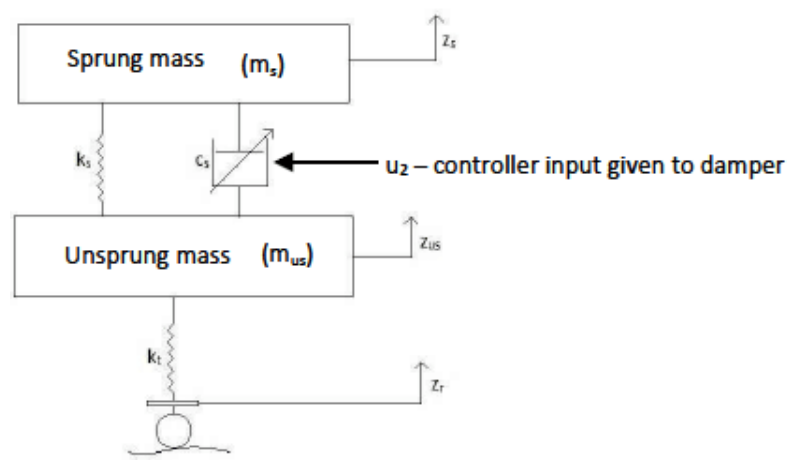

Figure 2: Quarter car model (Semi-active system)

In a conventional system, there is only a road input. But this paper incorporates damping coefficient as an input. So the quarter car model considered in this paper takes two inputs i.e., the road input and damping coefficient. This damping coefficient changes according to the PID controller used in the system. PID controller takes the input of sprung mass displacement and controller output is decided according to the criterion of minimizing sprung mass displacement (to improve ride comfort).

Given below are the state variables:

$x_{1}=z_{s}$

$x_{2}=z_{u s}$

$x_{3}=\dot{z}_{s}$

$x_{4}=\dot{z}_{u s}$

Given below are the state space equations:

$\dot{x}_{3}=\ddot{z}_{s}=\frac{k_{s}}{m_{s}}\left(x_{2}-x_{1}\right)+\frac{u_{2}}{m_{s}}\left(x_{4}-x_{3}\right)$

$\dot{x}_{4}=\ddot{z}_{u s}=\frac{k_{t}}{m_{u s}}\left(z_{r}-x_{2}\right)-\frac{k_{s}}{m_{u s}}\left(x_{2}-x_{1}\right)-\frac{u_{2}}{m_{u s}}\left(x_{4}-x_{3}\right)$

Where, $z_{s}$ - represents sprung mass displacement $z_{u s}-$ represents unsprung mass displacement $z_{r}$ - represents road input controller

\section{Results and discussion}

Simulink model of semi-active suspension system as well as passive suspension system was developed. The sprung mass acceleration of semi-active and passive suspension system was compared. The rattle space in the case of semi-active as well as passive suspension system was also compared.

As shown in figure $\mathbf{3}$, it depicts sprung mass acceleration of a Passive system, whereas figure $\mathbf{4}$ shows the sprung mass acceleration of a Semi-active system. In

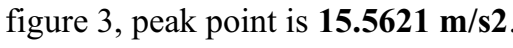

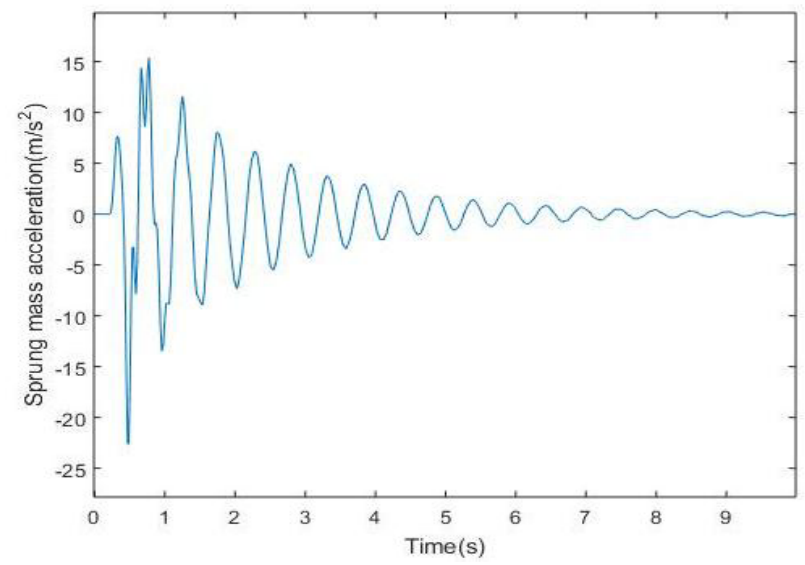

Figure 3: Sprung mass acceleration (Passive)

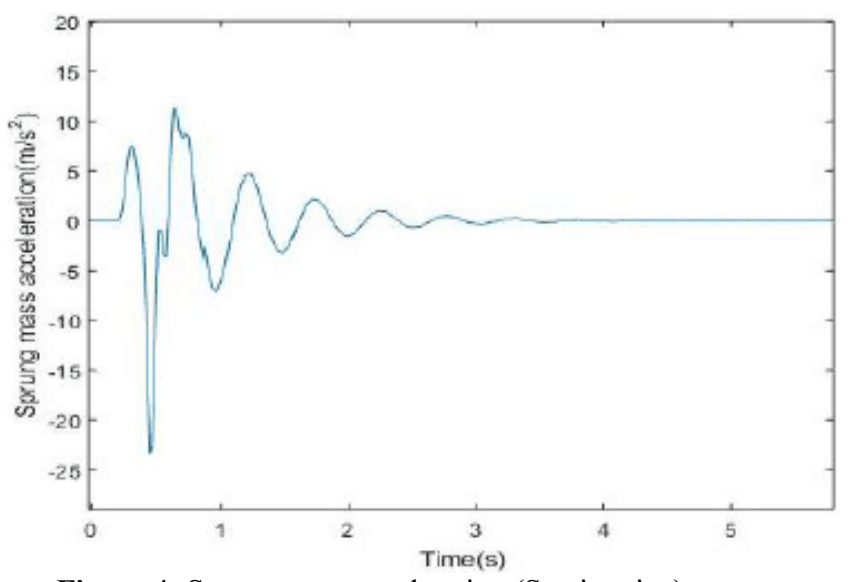

Figure 4: Sprung mass acceleration (Semi-active)

In figure 4 , the peak point is $\mathbf{1 1 . 2 7 6 7 ~} \mathbf{m} / \mathbf{s}^{\mathbf{2}}$. The given peak points show the highest vertical acceleration of sprung mass. So, semi-active system shows an improvement of $\mathbf{2 7 . 5 3 \%}$ over a passive system. Which ) means ride comfort is better in semi-active system. Also, sprung mass settles down in almost 4 seconds in semiactive system as compared to 10 seconds in passive system. Hence, semi-active suspension system provides a better ride comfort as compared to passive suspension system.

Rattle space is a relative displacement of sprung and unsprung mass. Figure 5 shows rattle space in passive system, whereas Figure $\mathbf{6}$ shows rattle space in semiactive system.

As shown below, in figure 5, highest displacement in $\mathrm{Y}$ direction is $\mathbf{0 . 1 2 1 0 5 m}$. 


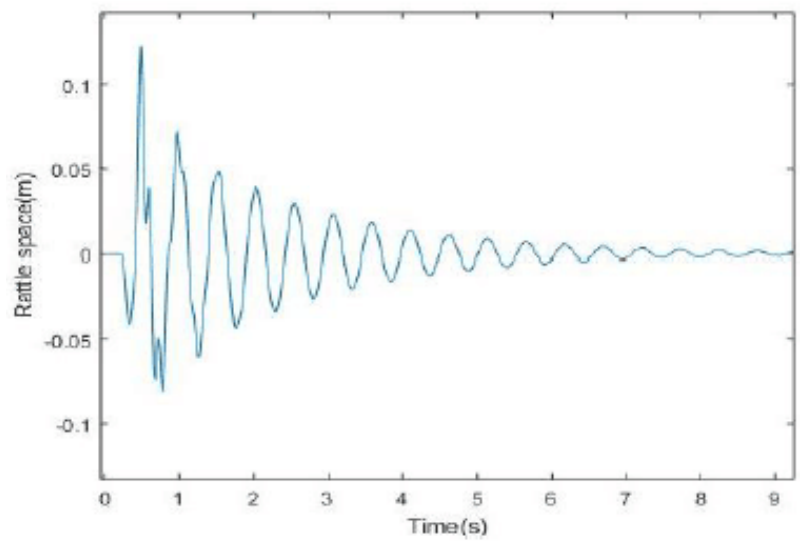

Figure 5: Rattle space (Passive)

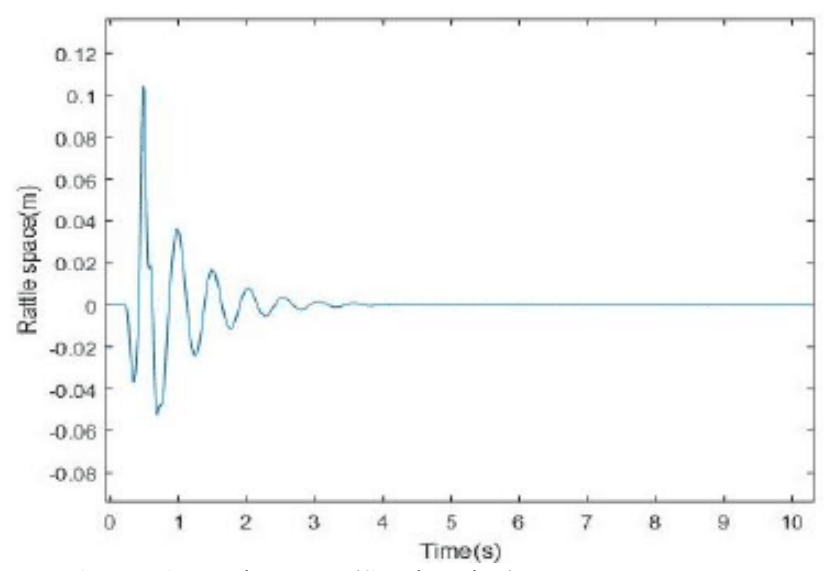

Figure 6: Rattle space (Semi-active)

In figure 6, highest displacement in $\mathrm{Y}$ direction is $\mathbf{0 . 1 0 4 4 8 m}$. So, semi-active system has lesser displacement as compared to passive system. Which means rider discomfort is less in semi-active system as compared to passive system. Also, the vehicle using semi-active system settles down in 4 seconds as compared to 10 seconds in vehicle using passive system. Hence there is an improvement of $\mathbf{1 3 . 7 \%}$.

\section{Conclusion}

The general approach in semi-actice suspension system is to control the damping constant using a controller. But it is considered as a constant in the mathematical model. However, this paper implements a novel approach to semi-active suspension system. The controller input (i.e., the damping coefficient) is considered variable and is given as input to the quarter car model. So there are two inputs in the system, first being the road input and second is the damping coefficient. Moreover, it has been found from the analysis that the semi-active suspension system provides a better ride comfort as compared to passive suspension system. And installing the controller helps achieve minimum acceleration quickly as compared to a normal semi-active suspension system.

The vibrations felt in Semi-active system are much less as compared to Passive system. And sprung mass acceleration can be directly correlated with ride comfort. So there has been a significant improvement in the ride comfort in a vehicle which uses Semi-active suspension system as compared to a vehicle which uses Passive suspension system.

Rattle space (relative displacement) is less in semiactive as compared to passive system, which can be concluded the simulation results. The improvement is significant. Since the settling time is relatively less in semi-active system, it provides much better ride comfort as compared to passive system.

\section{References}

1. A. Agharkakli, G. Shafiei Sabe, A. Barouz, International Journal of Engineering Trends and Technology Volume 3, 5 (2012)

2. N. H. Amer, R. Ramli, Wan Nor Liza Mahadi, Mohd. Azman Zainul Abidin , International Conference on Electrical, Control and Computer Engineering Pahang, Malaysia, June 21-22, 2011

3. Mohammad Meftahul Ferdaus, M M Rashid,Moinul Bhuiyan, Advanced Materials Research Vol. 939 (2014) Page - 615-622

4. M. Biglarbegian, W. Melek, F. Golnaraghi, Fuzzy Information Processing Society, 2006

5. T. Ram Mohan Rao, G. Venkata Rao, K. Sreenivasa Rao \& A. Purushottam, IJRRAS Vol. 5, 1 (2010)

6. B. Gadhvi, V. Savsani, Proceedings of the ASME 2014 International Mechanical Engineering Congress \& Exposition, Quebec, Canada, November 14-20, 2014

7. J. Patel, V. Savsani, R. Patel, ASME 2015 9th International Conference on Energy Sustainability collocated with the ASME 2015 Power Conference, the ASME 2015 13th International Conference on Fuel Cell Science, Engineering and Technology, and the ASME 2015 Nuclear Forum, June 2015.

8. A. Bagheri, M.J. Mahmoodabadi, H. Rostami, S. Kheybari, IJE TRANSACTIONS A: Basics Vol. 24, 3 (2011) Page - 291-300

9. S. Badran, A. Salah, W. Abbas, O. Abouelatta, Research Bulletin of Jordan ACM, Vol. 2, 8 (2012) Page - 42-51

10. A. Jamal Qazi, U. A. Farooqui, A. Khan, M. Tahir Khan, F. Mazhar, A. Fiaz, AASRI Conference on Intelligent Systems and Control (2013)

11. Y. Gandhi, V. Mehta, M. Patel, B. Gadhvi and A. Markana, Proceedings of the 2015 International Conference on Operations Excellence and Service Engineering (2015)

12. R.V. Rao, V.J. Savsani, D.P. Vakharia, ComputerAided Design, Vol. 43, 3 (2011)

13. T. Gillespie, Fundamentals of Vehicle Dynamics 